\title{
Anatomia do circuito arterial do encéfalo em Tamanduá-mirim
}

\author{
Anatomy of encephalon arterial circuit in lesser anteater
}

\author{
Ana Rita Lima ${ }^{\text {I* }}$ Luiza Corrêa Pereira ${ }^{I I}$ Érika Branco ${ }^{\mathrm{I}}$
}

\section{RESUMO}

\begin{abstract}
$O$ circuito arterial do encéfalo é de grande importância, pois é responsável pela vascularização do encéfalo, sendo este o principal órgão do sistema nervoso central e responsável por receber e processar informações. A espécie estudada foi escolhida por tratar-se de um mamífero pertencente à ordem Xenarthra e os integrantes dessa ordem são pouco estudados, devido à dificuldade de acesso a esses animais. Para desenvolver este trabalho, foram estudados cinco espécimes de Tamanduá-mirim (Tamandua tetradactyla). O material teve o sistema arterial injetado com látex Neoprene 650, corado em vermelho e fixado em solução aquosa de Formaldeído $10 \%$, sendo posteriormente dissecados para sistematização dos vasos. O circuito arterial da base do encéfalo do tamanduá-mirim circunda o corpo mamilar, a fossa interpenduncular, o túber cinéreo, a hipófise e o quiasma óptico. A porção rostral desse circuito é caracterizada pelos ramos rostrais das artérias carótidas internas e a porção caudal é constituída pelas artérias comunicantes caudais e ramos caudais da artéria carótida interna. Esta espécie animal apresenta o tipo II de irrigação encefálica no qual existe a participação das artérias carótidas internas e do sistema vértebro-basilar para a formação do circuito arterial.
\end{abstract}

Palavras-chave: anatomia, artérias da base do encéfalo, Tamandua tetradactyla.

\section{ABSTRACT}

The arterial circuit of the brain is of great importance because it is responsible for vascularization of the brain and this is the main organ of the central nervous system and responsible for receiving and processing information. The species was chosen because it is a mammal belonging to the
Xenarthra order and the members of this order are little studied because of the difficulty of access to these animals. To develop this work were studied five specimens of lesser anteater (Tamandua tetradactyla). The material had the arterial system filled with Neoprene latex stained in red, fixed in aqueous solution of $10 \%$ paraformaldehyde and dissected for vessels systematization. The encephalon arterial circuit of lesser anteater surrounds the mammillary body, the interpeduncular fossa, tuber cinereum, hypophysis and optic chiasm. The rostral portion of this circuit is characterized by rostral branches of internal carotid arteries and the caudal portion is formed by caudal communicant arteries from internal carotid artery. This animal species had a type II of encephalic irrigation with participation of internal carotid arteries and vertebrobasilar system to formation of arterial circuit.

Key words: anatomy, arteries of the base of the encephalon, Tamandua tetradactyla.

\section{INTRODUÇÃO}

O tamanduá-mirim (Tamandua tetradactyla) ocorre na América do Sul, a leste dos Andes, da Venezuela até o norte da Argentina e Uruguaio no Brasil, aparece em todos os biomas (Amazônia, Caatinga, Cerrado, Mata Atlântica, Pantanal e Pampa). Possui porte médio, cauda semi-preênsil e sem pelos longos, corpo coberto por pelos curtos, densos e grossos, apresentando coloração amarelada, em contraste com os pelos do dorso, com coloração escura, formando um desenho semelhante a um colete preto (TAVARES \& KOENEMANN, 2008).

'Laboratório de Pesquisa Morfológica Animal (LaPMA), Faculdade de Medicina Veterinária, Universidade Federal Rural da Amazônia (UFRA), Avenida Presidente Tancredo Neves, 2501, Bairro Montese, 66077-530, Belém, PA, Brasil. E-mail: vetlima@uol.com.br. *Autor para correspondência.

"Hydro - Mineração Paragominas S.A. Estrada Mineração, Paragominas, PA, Brasil. 
Pertence à ordem Xenarthra, apresentando comportamento e metabolismo letárgicos,o que se relaciona de forma direta ao seu hábito alimentar, constituído de alimentos pouco energéticos, tais como formigas, cupins, abelhas e mel. Para conseguir o alimento, ele utiliza suas fortes garras para fazer buracos no cupinzeiro e a língua para capturar os insetos (TAVARES \& KOENEMANN, 2008).

O estudo morfofuncional do sistema nervoso central vem demonstrando aspectos interessantes e pouco explorados, em especial aqueles relativos ao suprimento sanguíneo para o encéfalo (CUNHA et al., 2001). Nos animais domésticos, as artérias encefálicas apresentam diferentes arranjos, devido às artérias formadoras do "círculo de Willis", que consiste em um círculo arterial localizado na base do cérebro. Dessa forma, o comportamento das artérias encefálicas, comparativamente entre espécies, exibe um modelo básico ao qual são acrescentadas modificações relativas aos diferentes grupos de animais (LIMA et al., 2006).

O circuito arterial da base do encéfalo, interpretado por alguns autores (KRAMER, 1912; SHELLSHEAR, 1927; ROGERS, 1947) como uma anastomose de irrigação, foi analisado em alguns vertebrados, como o cavalo (SANTOS, 1987), fetos de bovinos azebuados (MELO, 1996); búfalos (DING et al., 2007); cães (ALCÂNTARA \& PRADA, 1992) raposas (DEPEDRINI \& CAMPOS, 2003); em ratos (ANDRADE, 1983) capivaras (RECKZIEGEL et al., 2004), marsupiais (LINDEMANN et al., 2000) e em perus (CARVALHO \& CAMPOS, 2011).

O conhecimento do circuito arterial encefálico tem sua importância ao subsidiar estudos para doenças como o acidente vascular encefálico ou derrame. Estudos vêm sendo desenvolvidos e o modelo ideal para pesquisas com acidente vascular encefálico deve envolver vários fatores e possuir um número de características similares aos humanos e, após a indução do processo patológico, os resultados devem ser investigados com o mínimo de limitações. Os modelos mais aplicáveis para pesquisas nesta área são os roedores e os lagomorfos. Tais modelos satisfazem todos os requerimentos básicos necessários para induzir, manipular e tratar as doenças que afetam os humanos. No entanto, outros modelos podem ser explorados por meio de estudos similares (CASALS et al., 2011).

$\mathrm{Na}$ tentativa de esclarecer aspectos da vascularização cerebral em Tamanduá-mirim, este estudo ateve-se à descrição dos vasos da base do encéfalo, determinando quais os vasos que contribuem para a formação do circuito arterial e seus principais ramos.

\section{MATERIAL E MÉTODOS}

Foram estudados cinco encéfalos de tamanduás-mirins, adultos, sendo três fêmeas e dois machos. Oriundos da empresa Hydro Mineração Paragominas - PA - S.A., sob autorização SEMA-PA no 455/2009 e 522/2009, doados ao Laboratório de Pesquisa Morfológica Animal (LaPMA) da Universidade Federal Rural da Amazônia (UFRA) após morte por atropelamento.

Foi realizada a injeção de látex Neoprene 650, corado com pigmento vermelho, utilizando como acesso a aorta torácica. Em seguida, as peças foram fixadas em solução aquosa de formoldeído a $10 \%$ por meio de injeções intramusculares, subcutâneas e intracavitárias. Os animais foram mantidos nessa solução por no mínimo $72 \mathrm{~h}$ e, decorrido este período, os encéfalos foram removidos. Toda a nomenclatura adotada foi baseada na Nomina Anatômica Veterinária (INTERNATIONAL COMMITTEE ON VETERINARY GROSS ANATOMICAL NOMENCLATURE, 2005).

\section{RESULTADOS}

Ao estudarmos os vasos da base do encéfalo do Tamandua tetradactyla, observamos que o circuito arterial nessa espécie foi suprido por três fontes, rostralmente pelas artérias carótidas internas e caudalmente pela artéria basilar (Figura 1).

Os arranjos dos ramos rostrais, direito e esquerdo, das artérias carótidas internas, apresentaram características morfológicas que lembram uma figura hexagonal, disposta ventralmente em relação a base do encéfalo, rostralmente ao quiasma óptico e, liberando ao longo de seu trajeto as artérias cerebral média e cerebral rostral e, ainda, a artéria comunicante rostral, que promoveu o fechamento do circuito arterial do encéfalo rostralmente (Figura 1).

A artéria cerebral média avançou lateralmente ao lobo piriforme e se distribuiu na superfície dorsolateral dos hemisférios cerebrais. A artéria cerebral rostral derivou-se medialmente da artéria carótida interna e projetou-se médio-rostralmente em direção ao sulco longitudinal onde se anastomosou com o vaso homólogo contralateral para formar a artéria comunicante rostral. Esta se apresentou como um vaso único, localizado rostro dorsalmente ao quiasma óptico, projetou-se em direção ao sulco longitudinal e supriu o corpo caloso, a área olfatória rostral e o pólo rostral dos hemisférios cerebrais (Figura 1).

A porção caudal do circuito arterial do encéfalo, formada pelos ramos caudais das artérias carótidas internas, em ambos os antímeros, originou- 

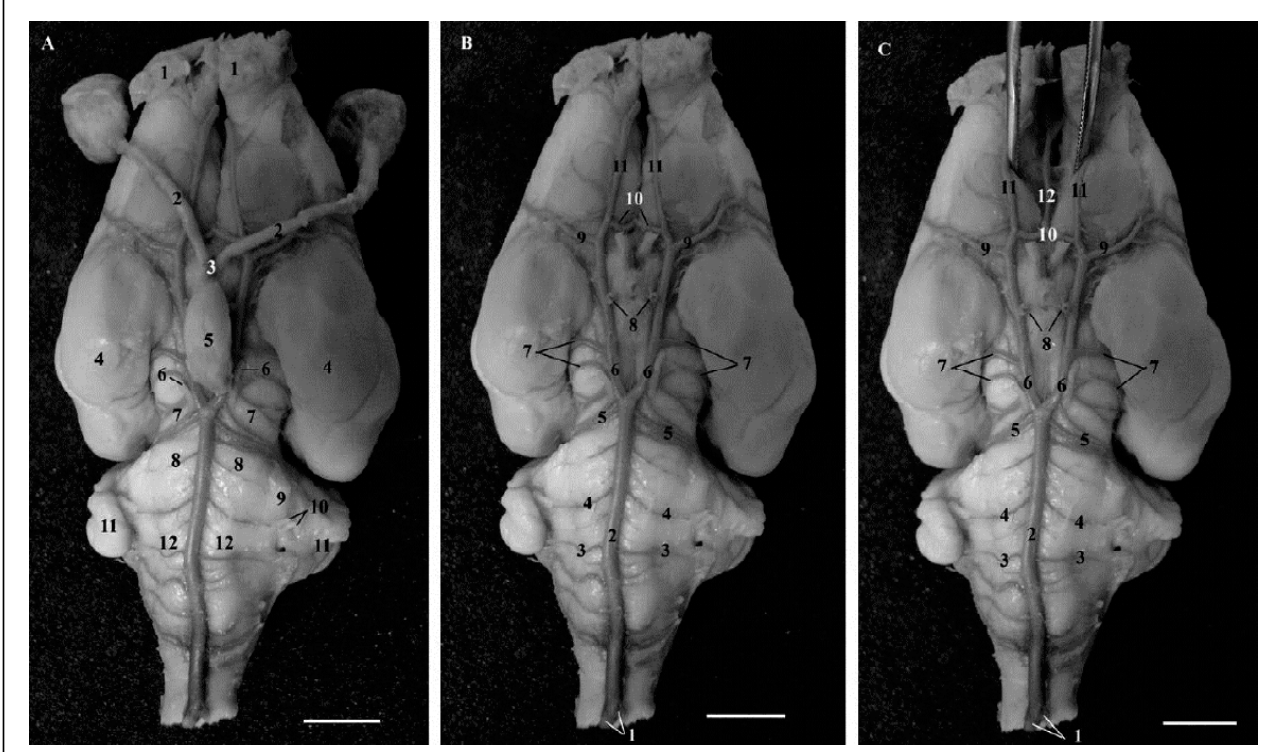

Figura 1 - Fotomacrografias do encéfalo de um Tamandua tetradactyla fêmea em que observamos as estruturas encefálicas em A: 1- Bulbo olfatório, 2- Nervo óptico, 3- Quiasma óptico, 4- Lobo piriforme, 5- Hipófise, 6- Nervo oculomotor, 7- Pedúnculos cerebrais, 8- Ponte, 9- Nervo trigêmeo, 10- Nervo facial e vestibulococlear, 11- Cerebelo e 12- Medula oblonga. Em B e C, observamos os vasos da base do encéfalo: 1- Artérias vertebrais, 2- Artéria basilar, 3- artéria cerebelar caudal, 4- artéria cerebelar média, 5- artéria cerebelar rostral, 6- artéria comunicante caudal, 7- artéria cerebral caudal, 8- artéria carótida interna, 9- artéria cerebral média, 10artéria cerebral rostral, 11- artéria etmoidal interna e 12- artéria comunicante rostral.

se das artérias homônimas e dirigiram-se, caudalmente, para terminarem, após as emergências das artérias cerebrais caudais, anastomosando-se nas superfícies ventrais dos pedúnculos cerebrais com os correspondentes ramos terminais da artéria basilar ou artérias comunicantes caudais (Figura 1).

As artérias vertebrais, direita e esquerda, originárias das artérias subclávias, ascenderam pelo pescoço e penetraram no canal vertebral após passarem pelos forames alar e vertebral lateral. Na superfície ventral da medula espinhal, esses vasos cruzaram o forame magno e se juntaram, formando a artéria basilar. A artéria basilar, após sua origem, dirigiu-se rostralmente e diminuiu progressivamente o seu calibre caudorrostralmente até a superfície ventral dos pedúnculos cerebrais, onde se bifurcou em seus ramos terminais ou artérias comunicantes caudais, que, por sua vez, anastomosaram-se com os correspondentes ramos caudais das artérias carótidas internas. Também foi possível observar a artéria basilar liberando ramos para a medula oblonga (Figura 1).

A artéria cerebelar caudal, originada no primeiro terço da artéria basilar, localizada em ambos os antímeros, dupla e disposta dorsalmente à medula oblonga, projetou-se lateralmente para irrigar a parte caudal e caudolateral dos hemisférios cerebelares. Já a artéria cerebelar média apresentou origem no terço médio da artéria basilar e se projetou lateralmente, cruzando as raízes dos nervos facial e vestibulococlear até alcançar a face dorsolateral dos hemisférios cerebelares. A artéria cerebelar média também emitiu ramos para a ponte. A artéria cerebelar rostral que, em todos os animais apresentou-se dupla no antímero direito e tripla no antímero esquerdo, localizou-se caudalmente na origem do nervo oculomotor (Figura 1).

Após a emissão dos ramos supramencionados, a artéria basilar bifurcou-se em seus ramos terminais ou artérias comunicantes caudais, que seguiram paralelas e passaram lateralmente e bem próximas do corpo mamilar e hipófise. A artéria basilar em seu trajeto caudo-rostral pelo sulco mediano apresentou sinuosidade discreta em todos os animais estudados (Figura 1).

Das artérias comunicantes caudais, surgiu a artéria cerebral caudal, dupla em ambos os antímeros em todos os espécimes estudados, avançou dorsolateralmente em torno do pedúnculo cerebral, atingindo o mesencéfalo e o pólo caudal dos hemisférios cerebrais (Figura 1). 


\section{DISCUSSÃO}

Estudos filogenéticos em animais domésticos demonstraram que diversos arranjos de múltiplas artérias constituem o círculo de Willis, mas essas diferentes características morfológicas não necessariamente representam as adaptações evolutivas (CASALS et al., 2011). Em vertebrados inferiores, somente a artéria carótida interna é responsável pela vascularização encefálica sem contribuição da artéria basilar. Em vertebrados superiores, somente a artéria basilar é responsável por essa função. Em uma terceira fase de evolução, a artéria basilar e a carótida são responsáveis pelo suprimento sanguíneo do encéfalo (CAMPOS et al., 2003).

De acordo com DE VRIESE (1905), a irrigação encefálica dos vertebrados pode ser classificada em três tipos: tipo I, em que a irrigação é exclusivamente via artéria carótida interna; tipo II, em que o encéfalo é irrigado pela carótida e sistema vértebro-basilar, com distribuição igual entre os dois sistemas ou a predominância de um sobre o outro; e o tipo III, em que a irrigação do encéfalo se dá exclusivamente via sistema vértebro-basilar. Exemplos dos três tipos incluem a galinha (Gallus gallus) (CAMPOS et al., 1995), gambá (Didelphis sp.) (VORIS, 1928; GILLIAN, 1972; LINDEMANN, et al., 2000) e a capivara (Hydrochoerus hydrochaeris) (RECKZIEGEL, et al., 2001).

De acordo com a classificação de DE VRIESE (1905), o tamanduá-mirim (Tamandua tetradactyla) apresenta o tipo II de irrigação encefálica, no qual existe a participação da artéria carótida interna e do sistema vértebro-basilar para a formação do circuito arterial. Esses dados corroboram o descrito para o cão (GETTY, 1986), gato (LIMAet al., 2006; LIMA et al., 2010), equino (GETTY, 1986; SOUZAet al., 2010), suíno (FERREIRA \& PRADA, 2005) e gambá (Didelphis sp.) (VORIS, 1928; GILLIAN, 1972; LINDEMANN, et al., 2000).

No cão, ALCÂNTARA \& PRADA (1992) descreveram que a figura formada pelas carótidas e artéria basilar não corresponde em seu todo nem a um polígono arterial, como descrito por BAPTISTA(1992), nem a um hexágono, como citam ELLENBERGER \& BAUM (1977), nem tampouco a um círculo arterial, como descreve DE VRIESE (1905). Já no Tamandua tetradactyla, a formação observada corresponde a um hexágono, tal como descrito por ELLENBERGER \& BAUM (1977).

Tratando da parte rostral do circuito arterial da base do encéfalo do Tamandua tetradactyla, entram nessa composição os ramos de divisão rostro-mediais derivados da artéria carótida interna direita e esquerda, sendo eles a artéria cerebral média, a artéria cerebral rostral e a artéria comunicante rostral. Esse tipo de conformação foi previamente descrito para o cão, equino e bovino (GETTY, 1986).

Com relação à parte caudal do circuito arterial da base do encéfalo do Tamandua tetradactyla, encontram-se nessa composição os ramos derivados da artéria basilar (artéria cerebelar caudal, cerebelar média e cerebelar rostral) e artérias comunicantes caudais (artéria cerebral caudal). Em perus (Meleagris gallopavo), o circuito arterial encefálico apresentouse aberto rostralmente e caudalmente e o suprimento de sangue para o encéfalo foi exclusivamente pelo sistema carotídeo (CARVALHO \& CAMPOS, 2011).

Nos animais domésticos, a artéria cerebelar caudal tem origem na artéria basilar, podendo ser múltipla, porém esta multiplicidade não ocorreu em nossos estudos. A artéria cerebelar rostral tem origem nas artérias comunicantes caudais e, ocasionalmente, nos suínos, ovinos e equinos, tem origem na artéria basilar, podendo ser múltipla em felinos, suínos, ovinos e equinos. A artéria cerebral caudal tem origem nas artérias comunicantes caudais, sendo dupla em equino e múltipla em caninos. Em nosso estudo, percebemos que a artéria cerebral caudal apresentou-se dupla em todos os espécimes analisados (SCHALLER, 1999).

Ainda alicerçado por DE VRIESE (1905), a artéria carótida interna é bem desenvolvida no embrião de rato, estando dividida em um ramo cranial e um ramo caudal, assim como em outros vertebrados. No início do desenvolvimento, as artérias vertebrais não existem, mas elas aparecem depois e se anastomosam com o ramo terminal caudal da artéria carótida interna. Em adultos, os ramos da artéria basilar se unem ao tronco da carótida, ocorre atrofia progressiva do sistema carótido cerebral caudal e um secundário e progressivo desenvolvimento do sistema vertebral. Considerando o arranjo das artérias cerebrais em vertebrados inferiores, em que o círculo de Willis é formado exclusivamente pelas artérias carótidas internas conectadas caudalmente a um sistema rudimentar da artéria vertebral, assume-se que as artérias que suprem o encéfalo são as artérias carótidas internas. Comparando com as artérias carótidas, as artérias vertebrais são o mais recente avanço na irrigação do cérebro.

De acordo com TANDLER (1898) e DE VRIESE (1905), a artéria basilar é formada pela anastomose das artérias vertebrais e subsequentemente bifurca ao nível da ponte em ramos terminais conhecidos como artérias comunicantes posteriores. DE VRIESE (1905) descreve ainda que a artéria cerebral caudal pertence morfologicamente ao 
grupo da carótida, e também pertence ao domínio vertebral, devido à sequência de mudanças vasculares secundárias que ocorrem em muitos roedores.

A artéria cerebral média em $\boldsymbol{C}$. cobaya é derivada do tronco comum das artérias cerebrais, enquanto que em $S$. vulgaris, S. aureogaster, $S$. americanus e Arctomys marmota, ela emerge da artéria comunicante caudal (TANDLER, 1898). Entretanto, DE VRIESE (1905) descreve que em $\boldsymbol{H}$. cristata, Lagostomus, Arctomys e Myoxus, a artéria cerebral média é derivada da artéria comunicante caudal, corroborando nossos resultados para o tamanduá-mirim, enquanto que, em Sciurus, C. cobaya e $\boldsymbol{C}$. aguti, ela é projetada como um ramo da bifurcação da artéria basilar.

A artéria cerebral rostral, descrita por TANDLER (1898) em C. cobaya, derivou do tronco comum da artéria comunicante caudal e, eventualmente, forma um círculo arterial fechado. De acordo com DE VRIESE (1905), em H. cristata, Lagostomus, Arctomys e Myoxus, as artérias cerebrais rostrais terminam nas artérias comunicantes caudais, que se juntam para formar a artéria comunicante rostral. No Tamandua tetradactyla, as referidas artérias originaram-se da artéria carótida comum e se unem para formar a artéria comunicante rostral.

As artérias cerebrais caudais direita e esquerda apresentaram-se duplas em todos os animais estudados e sempre localizadas na face ventral do pedúnculo cerebral, rostralmente ao nervo oculomotor. Esse achado discorda do que foi descrito por JENKINS (1978) em estudos anatômicos em cães, nos quais a artéria cerebral rostral apresentou-se única em ambos os antímeros.

\section{CONCLUSÃO}

A disposição e o arranjo apresentado pelas artérias da base do encéfalo do Tamandua tetradactyla são representados pelos ramos rostrais e caudais das artérias carótidas internas e, ainda, pelas artérias comunicantes caudais. A porção rostral desse circuito é caracterizada pelos ramos rostrais das artérias carótidas internas, sendo fechada pela presença da artéria comunicante rostral. A porção caudal do circuito arterial do encéfalo é constituída pelas artérias comunicantes caudais e ramos caudais da artéria carótida interna.

\section{REFERÊNCIAS}

ALCÂNTARA, M.A.; PRADA, I.L.S. Artérias da base do encéfalo de cães (Canis familiaris, L., 1758). Estudo anatômico de suas origens e comportamento. Brazilian Journal of Veterinary Research and Animal Science, v.33, p.67-71, 1992.
Disponível em: <http://www.scielo.br/scielo.php?pid=S141395962003000800004\&script=sci_arttext $>$. Acesso em: 29 maio, 2012. doi: $10.1590 /$ S1413-95962003000800004.

ANDRADE, F.F. $O$ circulo arterial da base do cérebro do rato de laboratório: contribuição para o seu estudo analítico e comparativo. 1983. 384f. Trabalho de síntese, no âmbito das provas de aptidão pedagógica e capacidade científica do Estatuto da Carreira Docente Universitária - Faculdade de Ciências Médicas da Universidade Nova de Lisboa, Lisboa.

BAPTISTA, B.V. Estudo comparado da circulação cerebral dos mamíferos domésticos e no homem - Razão de ser rede admirável. 1992. 89f. Tese (Doutorado em Anatomia) - Faculdade de Medicina do Rio de Janeiro, Rio de Janeiro, RJ.

CAMPOS, A. et al. Arteries at the base of the encephalon in horses. Occipitobasilar system. Brazilian Journal of Veterinary Research and Animal Science, v.40, p.107-117, 2003. Disponível em: <http://www.scielo.br/scielo.php?pid=S141395962003000800004\&script=sci_arttext $>$. Acesso em: 29 maio, 2012. doi: 10.1590/S1413-95962003000800004.

CAMPOS, R. et al. A systematic study of encephalic blood supply in Gallus gallus. I. Cerebral carotid arteries, collaterals and terminal branches, intercarotid anastomosis. Italian Journal of Anatomy and Embryology, v.100, p.111-121, 1995. ISSN 0004-0223.

CARVALHO, A.D.; CAMPOS, R. A systematic study of the brain base arteries in the turkey (Meleagris gallopavo). Pesquisa Veterinária Brasileira, v.31, n.1, p.39-46, 2011. ISSN 0100-736X. Disponível em: <http://www.scielo.br/scielo.php?script=sci_pdf\&pid=S0100736X2011001300007\&lng=pt\&nrm=iso\&tlng=en>. Acesso em: 29 maio, 2012.

CASALS, J.B. et al. The use of animal models for stroke research: a review. Comparative Medicine, v.61, n.4, p.305313, 2011. ISSN 1532-0820. Disponível em: <http:// www.ncbi.nlm.nih.gov/pmc/articles/PMC3155396/pdf/ cm2011000305.pdf>. Acesso em: 29 maio, 2012.

CUNHA, I.P. et al. Comportamento anatômico da artéria cerebelar média no cão (Canis familiaris - Linnaeus, 1758). Veterinária Notícias, v.7, n.2, p.13-22, 2001. ISSN 0104- 3463. Disponível em: <http://www.sovergs.com.br/conbravet2008/anais/cd/resumos/ R0583-2.pdf>. Acesso em: 29 maio, 2012.

DEPEDRINI, J.S.; CAMPOS, R. A systematic study of the brain base arteries in the Pampas fox (Dysicyon gymnocercus). Brazilian Journal of Morphological Science, v.20, n.3, p.181-188, 2003. ISSN 0102- 9010. Disponível em: <http:// www.jms.org.br /PDF/ v20n3a09.pdf>. Acesso em: 23 ago. 2011.

DE VRIESE, B. Sur la signification morphologique des artères cérébrales. Archives de Biologie, v.21, p.357-457, 1905. ISSN 0003-9624.

DING, Y. et al. The arterial supply to the brain of the Yak (Bos Grunniens). Annals of Anatomy, v.189, n.1, p.31-38, 2007. ISSN 0940-9602. Disponível em: <http:// www.sciencedirect.com/science>. Acesso em: 23 ago. 2011.

ELLENBERGER, W.; BAUM, H. Handbuch der vergleichenden anatomie der haustiere. Berlim: SpringerVerlag, 1977. 624p. 
FERREIRA, C.G.; PRADA, I.L.S. O circuito arterial da base do encéfalo em suínos (Sus scrofa domesticus Linnaeus, 1758), formação e comportamento. Brazilian Journal of Veterinary Research and Animal Science, v.42, n.1, p.5360, 2005. ISSN 1413-9596. Disponível em: <http:// www.fumvet.com.br/periodico/42/revista_42_1.pdf $>$. Acesso em: 23 ago. 2011.

GETTY, R. Sisson/Grossman anatomia dos animais domésticos. 5.ed. Rio de Janeiro: Guanabara Koogan, 1986. p.1513-1521.

GILLIAN, L. Blood supply to primitive mammalian brains. Journal of Comparative Neurology, v.145, p.209-222, 1972.

INTERNATIONAL COMMITTEE ON VETERINARY GROSS ANATOMICAL NOMENCLATURE. Nomina anatômica veterinaria. 5.ed. Hannover: Columbia, Gent, Sapporo: Editorial Committee, 2005. 166p.

JENKINS, T.W. Functional mammalian neuroanatomy. 2.ed. Philadelphia: Lea \& Febiger, 1978. 480p.

KRAMER, S.P. On the function of the circle of Willis. Journal of Experimental Medicine, v.15, p.348-355, 1912.

LIMA, E.M.M. et al. Estudo Anatômico das Artérias da Base do Encéfalo em Gatos (Felis catus domesticus). Ars Veterinaria, v.22, n.1, p.1-7, 2006. ISSN 0102-6380. Disponível em: <http://www.arsveterinaria.org.br/index.php/ars/ issue/view/6>. Acesso em: 23 ago. 2011.

LIMA, E.M.M. et al. Sistematização da origem, da distribuição e dos territórios da artéria cerebral caudal na superfície do encéfalo em gatos. Ciência Rural, v.40, n.9, p.1961-1965, 2010. ISSN 01038478. Disponível em: <http://www.scielo.br/scielo.php?pid=S0103$84782010000900017 \&$ script=sci_arttext $>$. Acesso em: 23 ago. 2011. doi: 10.1590/S0103-84782010000900017.

LINDEMANN, T. et al. A systematic study of brain Base arteries in the Opossum Didelphis albiventris. Brazilian Journal of Morphological Science, v.17, n.1, p.35-41, 2000. ISSN 0102-9010.

MELO, A.P.F. Estudo anatômico das artérias da base do encéfalo em fetos de bovinos azebuados. 1996. 106f. Dissertação (Mestrado em Ciências) - Faculdade de Medicina Veterinária e Zootecnia, Universidade de São Paulo, São Paulo, SP.
RECKZIEGEL, S.H. et al. Anatomy of the caudal cerebral artery on the surface of capybara (Hydrochoerus hydrochaeris) brain. Brazilian Journal of Morphological Science, v.21, n.3, p.131-134, 2004. ISSN 0102-9010. Disponível em: <http:/ /jms.org.br/PDF/v21n3a03.pdf>. Acesso em: 23 ago. 2011.

RECKZIEGEL, S.H. et al. A systematic study of the brain base arteries in capybara (Hydrochoerus hydrochaeris). Brazilian Journal of Morphological Science, v.18, n.2, p.103-110, 2001. ISSN 0102-9010. Disponível em: <http://jms.org.br/ PDF/v18n2a04.pdf>. Acesso em: 23 ago. 2011.

ROGERS, I.S. The function of the circulus arteriosus of Willis. Brain, v.70, n.2, p.171-178, 1947.

SANTOS, A. Estudo anatômico das artérias da base do encéfalo de eqüinos da raça puro sangue inglês. 1987. 111f. Dissertação (Mestrado em Ciências) - Faculdade de Medicina Veterinária e Zootecnia, Universidade de São Paulo, São Paulo, SP.

SCHALLER, O. Nomenclatura anatômica veterinária ilustrada. São Paulo: Manole, Brasil, 1999. 614p.

SHELLSHEAR, T.L. The arteries of the brain of the OrangUtan. Journal of Anatomy, v.61, n.2, p.167-197, 1927.

SOUZA, A.V.P. et al. Frequência da artéria caroticobasilar em eqüinos mestiços: estudo anatômico destinado a pesquisa experimental e ao diagnóstico por imagem. Pesquisa Veterinária Brasileira, v.30, n.8, p.685-688, 2010. ISSN 0100-736X. Disponível em: <http://www.scielo.br/pdf/pvb/ v30n8/13.pdf>. Acesso em: 23 ago. 2011.

TANDLER, J. Zur vergleichenden anatomie der kopfarterien bei den mammalia. Denkschr Akademie Wissenchaften, v.67, p.677-689, 1898.

TAVARES, S.V.; KOENEMANN, J.G. Ocorrência de Tamandua tetradactyla (Linnaeus, 1758) (Xenarthra, Myrmecophagidae) no Município de Itaqui, Fronteira Oeste do Rio Grande do Sul, Brasil. Biodiversidade Pampeana, v.6, n.2, p.30-33, 2008. ISSN 16796179. Disponível em: <http://revistaseletronicas.pucrs.br/ojs/ index.php/biodiversidadepampeana/article/viewFile/4878/3810>. Acesso em: 23 ago. 2011.

VORIS, H.C. The arterial supply of the brain and spinal cord of the Virginian opossum (D. virginiana). Journal of Comparative Neurology, v.44, p.403-423, 1928. 